\title{
Expression of integrin-linked kinase improves cardiac function in a swine model of myocardial infarction
}

\author{
WEN LU ${ }^{1}$, JUN XIE ${ }^{2}$, RONG GU ${ }^{2}$ and BIAO XU ${ }^{2}$ \\ ${ }^{1}$ Department of Cardiology, Xuzhou Central Hospital, Xuzhou, Jiangsu 221009; ${ }^{2}$ Department of Cardiology, Nanjing Drum \\ Tower Hospital, The Affiliated Hospital of Nanjing University Medical School, Nanjing, Jiangsu 210008, P.R. China
}

Received October 3, 2016; Accepted December 21, 2016

DOI: $10.3892 /$ etm.2017.4162

\begin{abstract}
Previous studies have described the beneficial effects of overexpressing integrin-linked kinase (ILK) after myocardial infarction (MI) in small animal models. However, the effects of ILK in pre-clinical large animals are not known. To move closer to clinical translation, we examined the effects of ILK gene transfer in a swine model of ischemic heart disease. Swine received percutaneous intracoronary injections of adenoviral vector expressing ILK $(n=10)$ or empty ad-null $(n=10)$ in the left anterior descending coronary artery (LAD) following LAD occlusion. Four weeks after transfection, we confirmed that transgene expression was restricted to the infarcted area in the cardiac tissue. Imaging studies demonstrated preserved cardiac function in the ILK group. ILK treatment was associated with reduced infarcted scar size and preserved left ventricular (LV) geometry (LV diameter and LV wall thickness). Enhanced angiogenesis was preserved in the ILK animals, along with reduction of apoptosis. ILK gene therapy improves cardiac remodeling and function in swine following MI associated with increased angiogenesis, reduced apoptosis, and increased cardiomyocyte proliferation with no signs of toxicity. These results may deliver a new approach to treat post-infarct remodeling and subsequent heart failure.
\end{abstract}

\section{Introduction}

Despite advances in pharmacology, interventional cardiology techniques, and devices and surgery, heart failure (HF) remains the leading cause of mortality and hospitalization worldwide (1). Cardiac remodeling is generally accepted as a determinant of the clinical course of HF. Following myocardial infarction (MI), cardiomyocyte loss and increased load

Correspondence to: Dr Biao Xu, Department of Cardiology, Nanjing Drum Tower Hospital, The Affiliated Hospital of Nanjing University Medical School, 321 Zhong Shan Road, Nanjing, Jiangsu 210008, P.R. China

E-mail: gkjmomgnjjglpm5@163.com

Key words: myocardial infarction, remodeling, gene therapy, integrin-linked kinase trigger gene expression changes, result in molecular, cellular, and interstitial changes that manifest clinically in changes in size, shape, and function of the heart $(2,3)$. Preventing or attenuating the signaling pathways leading to changes in heart size is an important goal of anti-remodeling therapy (4).

A target recently identified in the heart remodeling pathway is integrin-linked kinase (ILK), a serine/threonine protein kinase widely expressed in mammalian tissues that plays important roles in transducing cell-matrix interaction-induced biomechanical signals, including cytoskeleton remodeling, angiogenesis, cell growth, proliferation, survival, and differentiation $(5,6)$. Indeed, mutations in ILK have been reported in human patients with dilated cardiomyopathy (DCM) (7) and targeted ILK deletion in the murine heart causes spontaneous DCM and HF (8). In addition, ILK controls the recruitment of endothelial progenitor cells to the ischemic tissue (9) and ILK gene therapy improves cardiac remodeling and function in rats after MI (5).

The promising results in rodents led us to conduct further investigation on the therapeutic effects of ILK in a large animal model that is closer to approximate human physiology and anatomy. In the present study, we investigated whether heart transfection of ILK following acute MI in swine showed the same therapeutic effects as those described in mice and rats. The present study focused on the mechanisms of ILK action and the safety of ILK intervention.

\section{Materials and methods}

Recombinant adenovirus construction. Recombinant adenoviral vector carrying human wild-type ILK together with humanized recombinant green fluorescent protein (hrGFP) cDNA (ad-ILK) or only hrGFP (ad-null) under the control of the CMV promoter was prepared as previously described (10).

Animal model of MI and adenoviral vector delivery. Animal experiments were performed following the guidelines in the Guide for the Care and Use of Laboratory Animals published by the National Institutes of Health (National Institutes of Health publication No. 85-23, revised 1985). The procedures were approved by the Institutional Animal Care and Use Committee of Nanjing Drum Tower Hospital.

MI was induced as described previously (11). Briefly, 20 mini pigs (15-17.5 kg) were randomly divided into two 
groups that received ad-ILK $(n=10)$ or ad-null $(n=10)$. The mini pigs were pre-medicated using intramuscular ketamine. After the intravenous line was placed, mini pigs were intubated and ventilated with $100 \%$ oxygen (oxygen flow was 3 1/min). Electrocardiograms were monitored continuously during the operation. A 7F femoral artery sheath was then advanced to the left coronary artery ostium. After confirming catheter positioning by coronary angiogram, a coronary angioplasty balloon was placed in the proximal left anterior descending coronary artery (LAD) to the first diagonal branch and pre-conditioned for $30 \mathrm{sec}$ for three times followed by $90 \mathrm{~min}$ reperfusion (the balloon was inflated to $4 \mathrm{~atm}$ ). Then, $2 \times 10^{9}$ viral particles of ad-ILK or ad-null were delivered by anterograde intracoronary infusion over $10 \mathrm{~min}$. The MI model was confirmed by observing a substantial ST-segment elevation and by serum cTnT levels six hours after surgery.

Ultrasonic cardiogram. Transthoracic echocardiography was assessed before inducing MI and four weeks after gene delivery. After general anesthesia, all the measurements were performed under repeated, short end-expiratory breath holds. Transthoracic 2D echocardiography was performed with a dynamic focused $3.5-55 \mathrm{MHz}$ probe and using SONO 5500 ultrasound system (Philips, Eindhoven, The Netherlands). Left ventricular (LV) end-systolic diameter, LV end-diastolic diameter, interventricular septal thickness in diastole (IVSD), LV posterior wall thickness (LVPW), and LV ejection fraction (LVEF) were measured.

Positron emission tomography (PET). Regional myocardial PET was evaluated four weeks after gene transfer as previously described with minor modifications. Before PET scans, pigs were maintained under general anesthesia and blood glucose ranged from 5 to $6 \mathrm{mmol} / \mathrm{l}$. Then the animals were placed in the dorsal (supine) position, and PET scans were performed $60 \mathrm{~min}$ after intravenous injections of $2.65 \mathrm{MBq}$ of 18F-fluorodeoxyglucose per kilogram. PET images were acquired in 2D mode. Transaxial cardiac images were then reconstructed into horizontal short-axis, as well as horizontal and vertical long-axis with a $4 \mu \mathrm{m}$ thickness. Delayed PET scans were performed if necessary. Three axis views were analyzed further.

Single-photon emission computed tomography (SPECT). Four weeks after gene transfer, $99 \mathrm{mTc}$-sestamibi of $1 \mathrm{MBq}(10 \mathrm{mCi})$ per kilogram was delivered to the animals. After $40 \mathrm{~min}$, the heart images were collected with a dual-head $\gamma$ camera (Skylight, Philips) in step-and-shoot mode. Then, 64 images (64x64 matrix) were acquired, $40 \mathrm{sec}$ each and throughout a $180^{\circ}$ arc. The images were reconstructed along the short, horizontal long, and vertical long axes of the heart. Quantitative analysis of perfusion defects was performed according to the AHA procedural guidelines for myocardial perfusion imaging in nuclear cardiology (12).

Histology. At the end of the study, pigs were euthanized by injecting $20 \mathrm{mmol}$ potassium and their hearts were sectioned into six sections. Hearts, liver, and kidneys were fixed with formalin, embedded in paraffin for subsequent histological, immunohistochemical, and TUNEL examination. Tissue samples collected for western blot analysis were snap-frozen with liquid nitrogen.

Immunohistochemistry. To differentiate between the border and infarct area, the frozen heart tissue was first stained with hematoxylin and eosin (H\&E). To measure microvessel density, the frozen sections $(5 \mu \mathrm{m})$ were stained with anti-rat vWF antibody (1:200, BD Laboratory), followed by goat anti-rabbit secondary antibody conjugated with EnVision HRP/DAB (Dako, Carpinteria, CA, USA). Microvessel density was calculated as previously reported (13).

Transgene expression and TUNEL assay. The sections were incubated with mouse anti-rabbit sarcomeric actin (1:50; Abcam, Cambridge, MA, USA) and rabbit anti-GFP (1:100; Beyotime Institute of Biotechonolgy, Beijing, China). Alexa Fluor 488 goat anti-mouse secondary antibody (1:200) and Alexa Fluor 555 goat anti-rabbit secondary antibody (1:250) (both from Molecular Probes, Inc., Eugene, OR, USA) were applied for subsequent detection. Apoptosis was assessed by TUNEL assay on slides of myocardium using the DeadEnd Fluorimetric TUNEL System (Promega Corp., Madison, WI, USA). The apoptotic cells were measured by counting the TUNEL-positive nuclei relative to the total number of nuclei counterstained with 4',6-diamidino-2-phenylindole (Sigma, St. Louis, MO, USA).

Western blot analysis. Western blotting was conducted as previously described (14). Rabbit monoclonal ILK antibody (dilution, 1:500; cat. no. ab52480; Abcam, Cambridge, MA, USA), rabbit monoclonal GFP antibody (dilution, 1:1,000; cat. no. 2037S) and rabbit monoclonal GAPDH antibody (dilution, 1:1,000; cat. no. 3683) (both from CST, Beverly, MA, USA) were used. Protein band densities and were calculated by Image J followed by normalization using GAPDH as the internal control.

Statistical analysis. Continuous variables were reported as mean \pm standard error of the mean. Data analysis was performed using SPSS 20 (IBM SPSS, Armonk, NY,USA). The unpaired Student's t-test was used between group differences if normal distribution was assumed or Mann-Whitney U test when distributions were not normal. ANOVA was used to test for differences among at least three groups. $\mathrm{P}<0.05$ was considered to indicate a statistically significant difference.

\section{Results}

Large-animal model of MI. Percutaneous, catheter-based intermittent balloon occlusion of the proximal LAD (Fig. 1A) resulted in reproducible perfusion defects of the anterior wall and MI. The infarcted pigs were confirmed by a substantial ST-segment elevation (Fig. 1B-D), the detection of serum cTnT $6 \mathrm{~h}$ after surgery (Fig. 1G), and pathological H\&E staining after sacrificing the animals (Fig. $1 \mathrm{H}$ and I). These results supported the successful induction of MI in mini pig that we can use to study the therapeutic effects of ILK.

ILK gene therapy increases cardiac ILK expression. Following the injection of adenoviral particles, gene transfer 

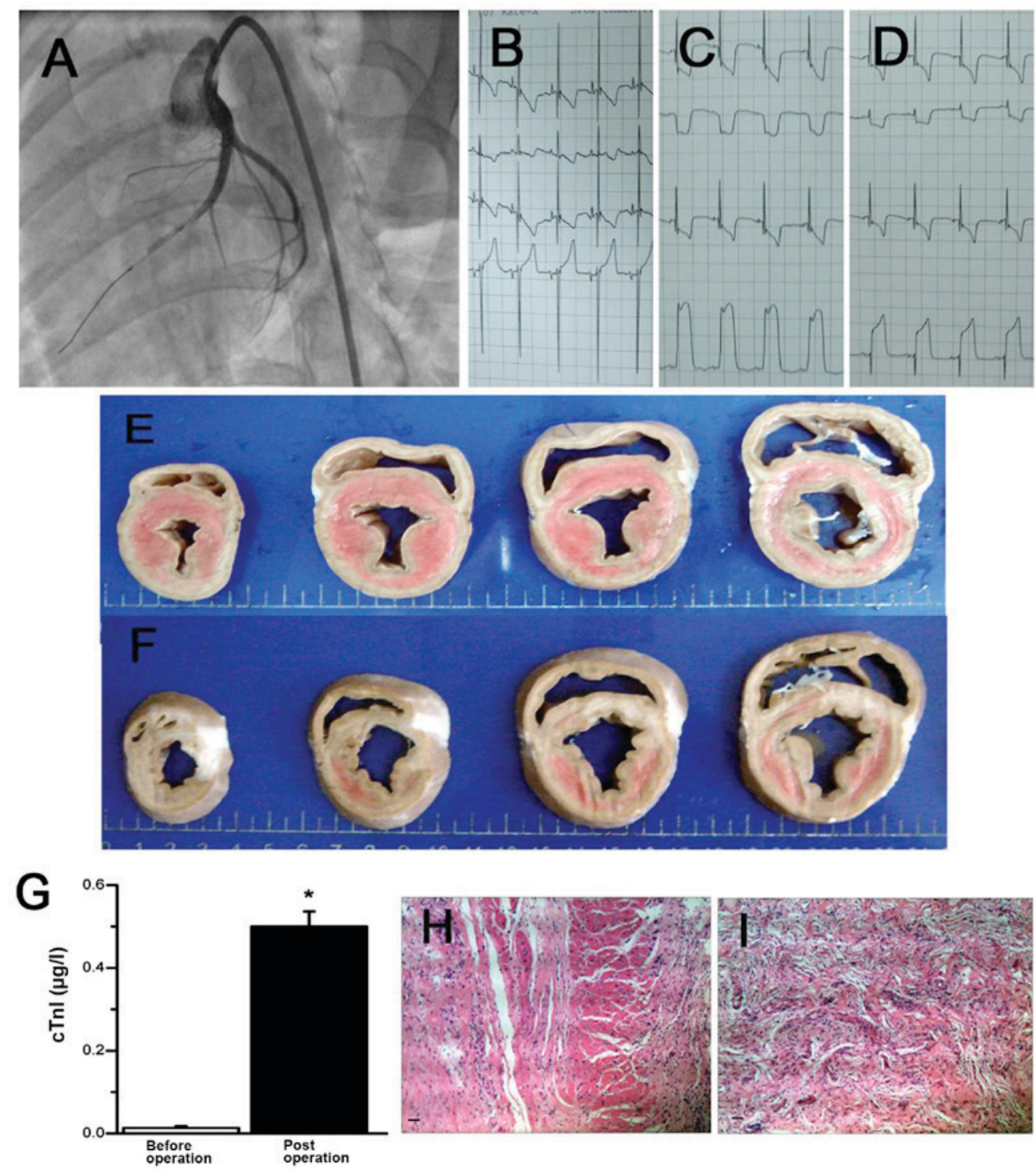

Figure 1. Establishing a swine myocardial infarction model. (A) Pigs underwent 90 min proximal left anterior descending coronary artery balloon occlusion. (B) Preoperative ECG. (C) ST elevation in operation. (D) ECG of post-operation. (E) Heart slices of sham-operation. (F) Heart slices of infarct area with white scar. (G) Serum cTnI levels of pre- and post-surgery, ${ }^{*}$ compared with before operation, $\mathrm{P}<0.01$. (H and I) Heart section staining by H\&E indicated cardiomyocyte necrosis. Scale bars, $100 \mu \mathrm{m}$.

was confirmed by detecting the expression of ILK and/or hrGFP. First, we detected in situ transgene expression derived from adeno-virus. For this, we stained heart sections from MI animals four weeks after transfection for $\alpha$-sarcomeric actin, a marker of cardiomyocytes (Fig. 2A), and hrGFP. GFP-positive cells were found in the infarction zone (Fig. 2B). In addition, the majority of hrGFP-positive cells were overlaid with $\alpha$-sarcomeric actin (Fig. 2C), indicating successful ad-ILK transfection in cardiomyocyte, although some cardiac fibroblasts appeared hrGFP-positive.

For quantitative studies, we detected the exogenous expression of transfected ILK using western blot analysis. Four weeks after adenoviral delivery, the expression of ILK in the infarcted zone was significantly elevated in the ad-ILK group relative to the ad-null controls by western blot analysis (Fig. 2D, $\mathrm{E}$ and $\mathrm{G} ; \mathrm{P}<0.05)$. However, we found no differences in ILK levels in the infarction remote area (Fig. 2F-H; $\mathrm{P}>0.05$ ). These results confirmed that the expression of ILK from the viral vector was localized to the infarcted site.
ILK gene rescues cardiac function and preserves myocardial perfusion. After detecting positive expression of ILK from the viral transfection, we performed cardiac functional studies with echocardiography (Fig. 3A-D). Four weeks after MI, two cases of apical aneurysm occurred in ad-null group, whereas no cases occurred in the ad-ILK group. ILK overexpression exhibited a further improvement in percentage of $\mathrm{EF}(56.03 \pm 3.19 \%)$ compared to controls $(36.70 \pm 2.68 \%, \mathrm{P}<0.05)$ (Fig. 3A). Moreover, interventricular septum thickness (IVSd) improved in the ad-ILK group $(5.48 \pm 0.87 \mathrm{~mm})$ compared to the ad-null group $(4.17 \pm 0.74 \mathrm{~mm}, \mathrm{P}<0.05)$ (Fig. 3B). Percent fractional shortening (\% FS) also demonstrated significant improvement in the ad-ILK group $(46.23 \pm 2.35 \mathrm{~mm})$ compared to the ad-null group $(37.31 \pm 1.98 \mathrm{~mm}, \mathrm{P}<0.05)$ (Fig. $3 \mathrm{C}$ ). By contrast, the LVPW showed no significant difference between the two groups. These data suggested improved cardiac function four weeks after adenoviral delivery of ILK.

SPECT is a commonly available direct method for assessing myocardial cell viability (15). We used SPECT to 


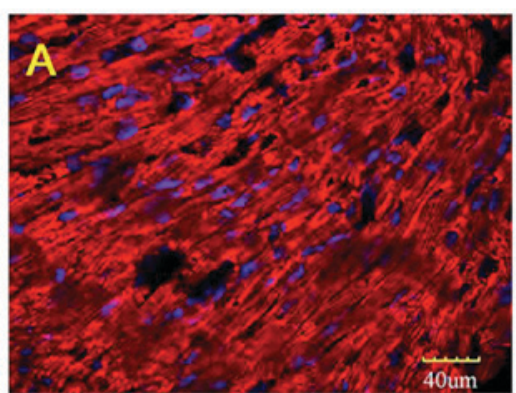

D
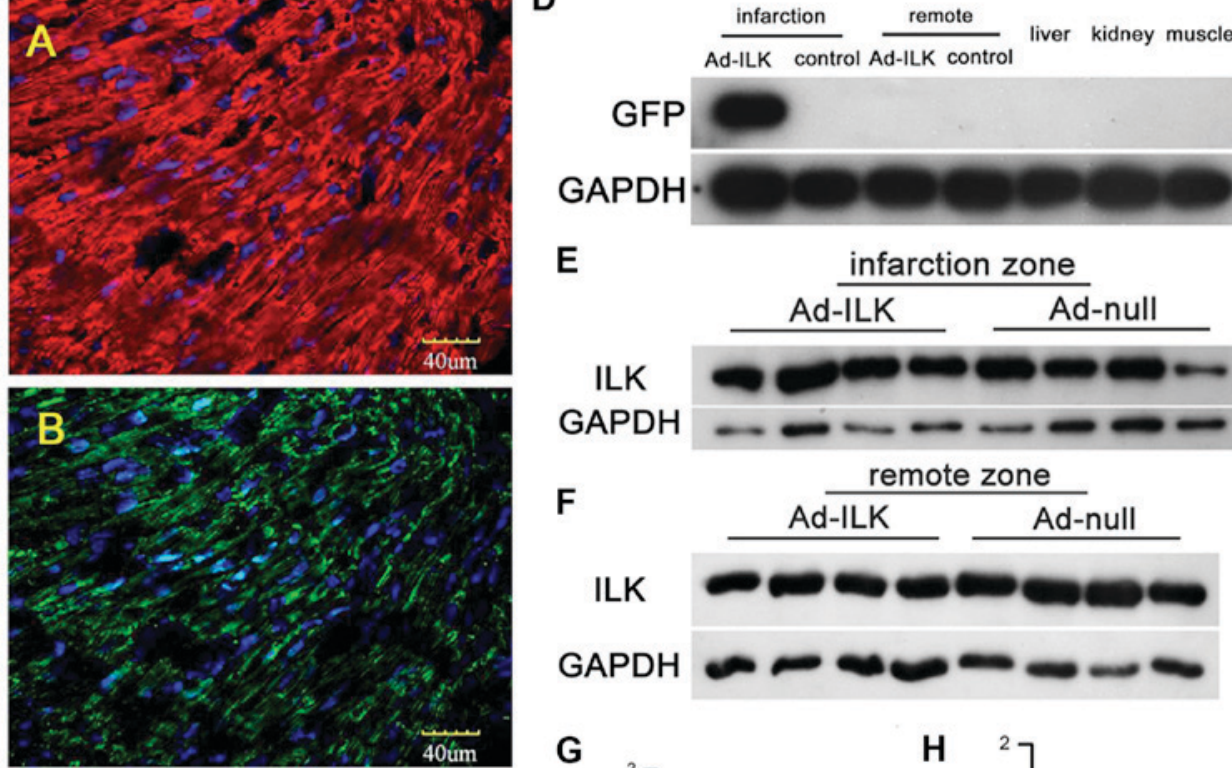

E
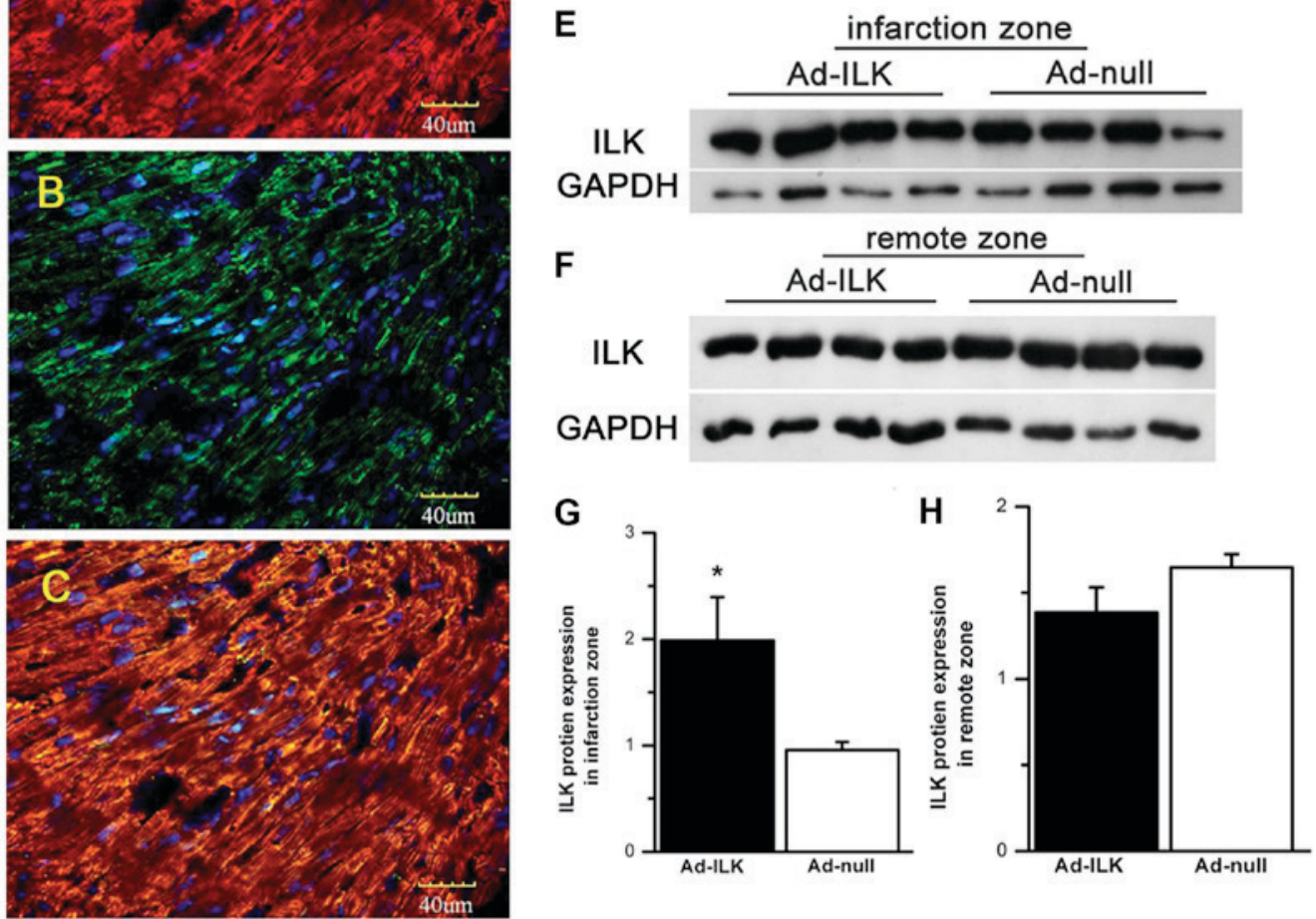

Figure 2. Expression of ILK and hrGFP four weeks after adenoviral delivery. (A) Immunofluorescence for $\alpha$-actin and DAPI in cardiomyocytes (B) Immunofluorescence for hrGFP expression in the myocardium. (C) hrGFP expression appeared in $\alpha$-actin-positive cells, indicating successful Ad-ILK transfection into cardiomyocyte. (D) Western blotting showing hrGFP expression in the infarction zone four weeks after adenoviral delivery compared with null control. GAPDH expression is shown as internal control. (E and G) Western blotting showing ILK expression after adenoviral delivery in the infarction zone compared with null control. ( $\mathrm{F}$ and $\mathrm{H}$ ) Western blotting showing ILK expression after adenoviral delivery in the infarction remote zone compared with null control. GAPDH expression is shown as internal control. "P>0.05, $\mathrm{n}=6$. Scale bars, $40 \mu \mathrm{m}$. ILK, integrin-linked kinase; hrGFP, humanized recombinant green fluorescent protein; DAPI, 4',6-diamidino-2-phenylindole.

determine whether ad-ILK delivery can attenuate the severity of myocardial ischemia. The extent of rest/stress perfusion defect was markedly smaller when ILK was overexpressed compared with the control (SRS $8.25 \pm 5.7$ vs. $14 \pm 3.12$, $\mathrm{P}<0.05$ ) (Fig. 3E and F), further supporting the beneficial effect of ILK.

Compared to SPEPCT, PET-CT possesses the advantage measuring cardiomyocytes metabolism quantitatively (16). In the present study, PET images obtained four weeks after ILK treatment displayed improved levels of myocardial metabolism compared to control. Then, we combined the PET and SPECT images and the infarcted zones were determined according to the calculation of DS scores, a sensitive index that discriminates the vitality of the ischemic myocardium. The DS scores for the ad-ILK group were highly significant, indicating the improved vitality of myocardium when treating with ad-ILK (Fig. 3E and F).

ILK gene therapy increases microvessel density and reduces apoptosis in the infarct zone. vWF staining four weeks after MI demonstrated that ad-ILK delivery increased microvessel density in the peri-infarct myocardium (136.02 \pm 6.32$)$ compared to the ad-null group $(91.56 \pm 7.81$,
$\mathrm{P}<0.01$ ) (Fig. 4C and D). TUNEL assay was conducted to quantify cardiomyocyte apoptosis following the infarction. The percentage of TUNEL-positive cardiomyocytes was markedly reduced in the ad-ILK group $(0.57 \pm 0.37 \%)$ compared to the ad-null group $(1.01 \pm 0.93 \%, \mathrm{P}<0.01)$ in the infarct zone four weeks post-MI (Fig. 4A and B). These studies confirmed the protective effect of ILK by promoting microvessel density and preventing cardiomyocyte cell death.

\section{Discussion}

The present preclinical study provides evidence for the benefits for ILK transfer on cardiac function after MI caused by a transient coronary occlusion in mini pigs. Four weeks after gene transfer, the ILK treatment group exhibited reduced infarct scar size, preserved LV geometry, enhanced angiogenesis, decreased apoptosis, and increased cardiomyocyte proliferation. These results support previous work in rodent models on the protective activity of ILK during MI.

The argument of gene transfection efficiency arises due to the paradox of time selection for gene transfer. Post-infarction remodeling has been arbitrarily divided into an early phase (within 72 h) and a late phase (beyond 72 h) (17). Currently, 

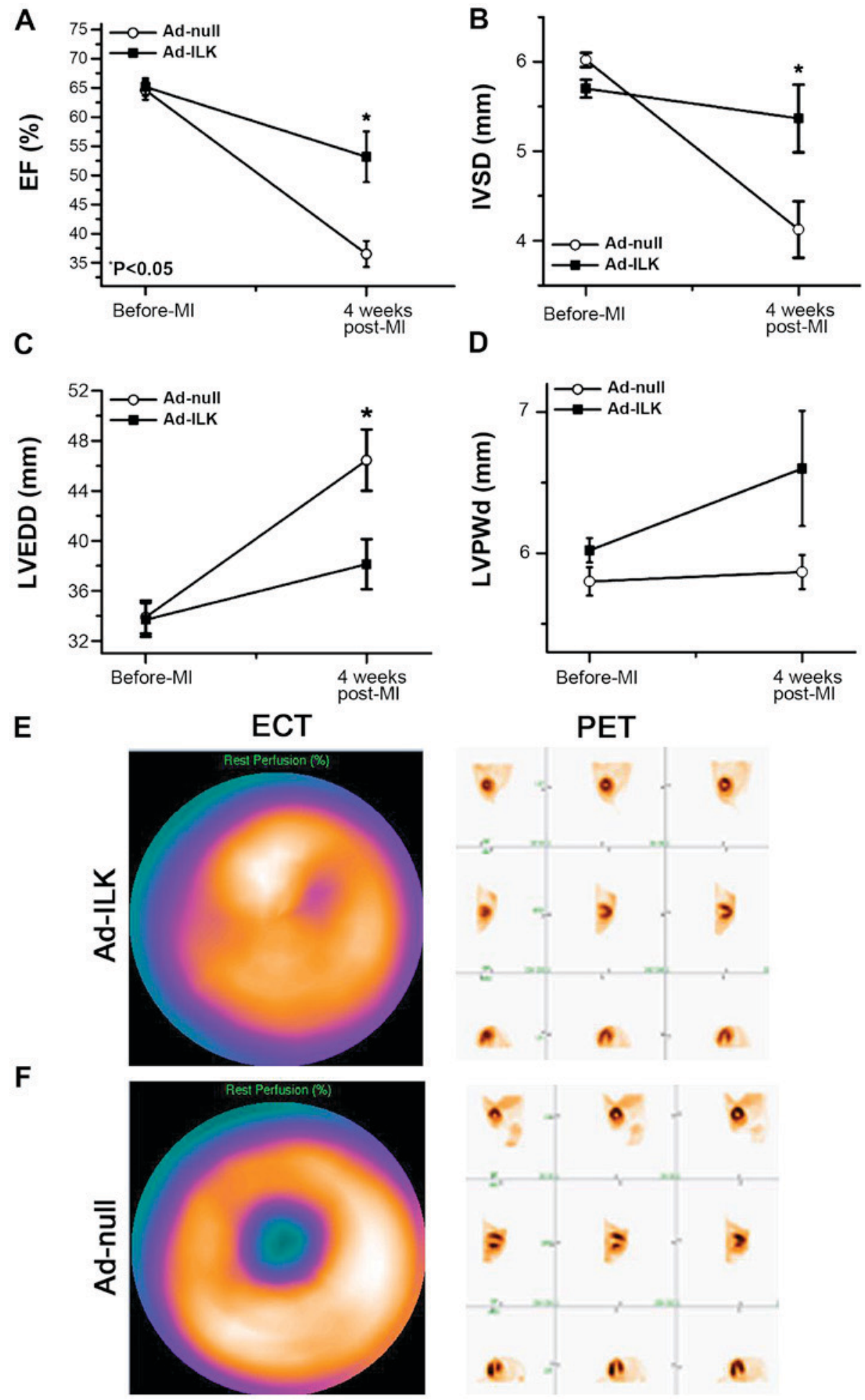

Figure 3. Heart imaging studies in mini pig MI. (A-D) LVEDD, IVSD, EF and LVPWd improved in the ad-ILK group compared with the ad-null group. n=6, ${ }^{*} \mathrm{P}<0.05$. (E and F) Ad-ILK improves heart function four weeks post-MI. The myocardial perfusion image by ECT and the myocardial metabolic image by PET. LVEDD, LV end-diastolic diameter; IVSD, interventricular septal thickness in diastole; MI, myocardial infarction; PET, positron emission tomography.

most preclinical gene transfer studies have focused on the chronic ischemic heart disease (a late phase of remodeling) (18). However, fewer studies address gene transfer on acute MI (an early phase) model. The early phase is associated with the expansion of the infarct zone and this time is critical to normalize several key molecules during the early phase of MI (17). Substantial evidence supports the effectiveness of emergency percutaneous coronary intervention (PCI) in acute MI (19).
ILK transfer can be conducted concurrently with emergency PCI, which is available in clinical practice. Recombinant adenoviruses can express physiologically significant levels of transgene starting 2-4 h after delivery (20). Thus, in the present study, we delivered ILK immediately after inducing MI in the animal model. Aneurysms form during the early remodeling phase (17) and we detected them only in the ad-null group, partly indicating the benefits of early phase transfer. 

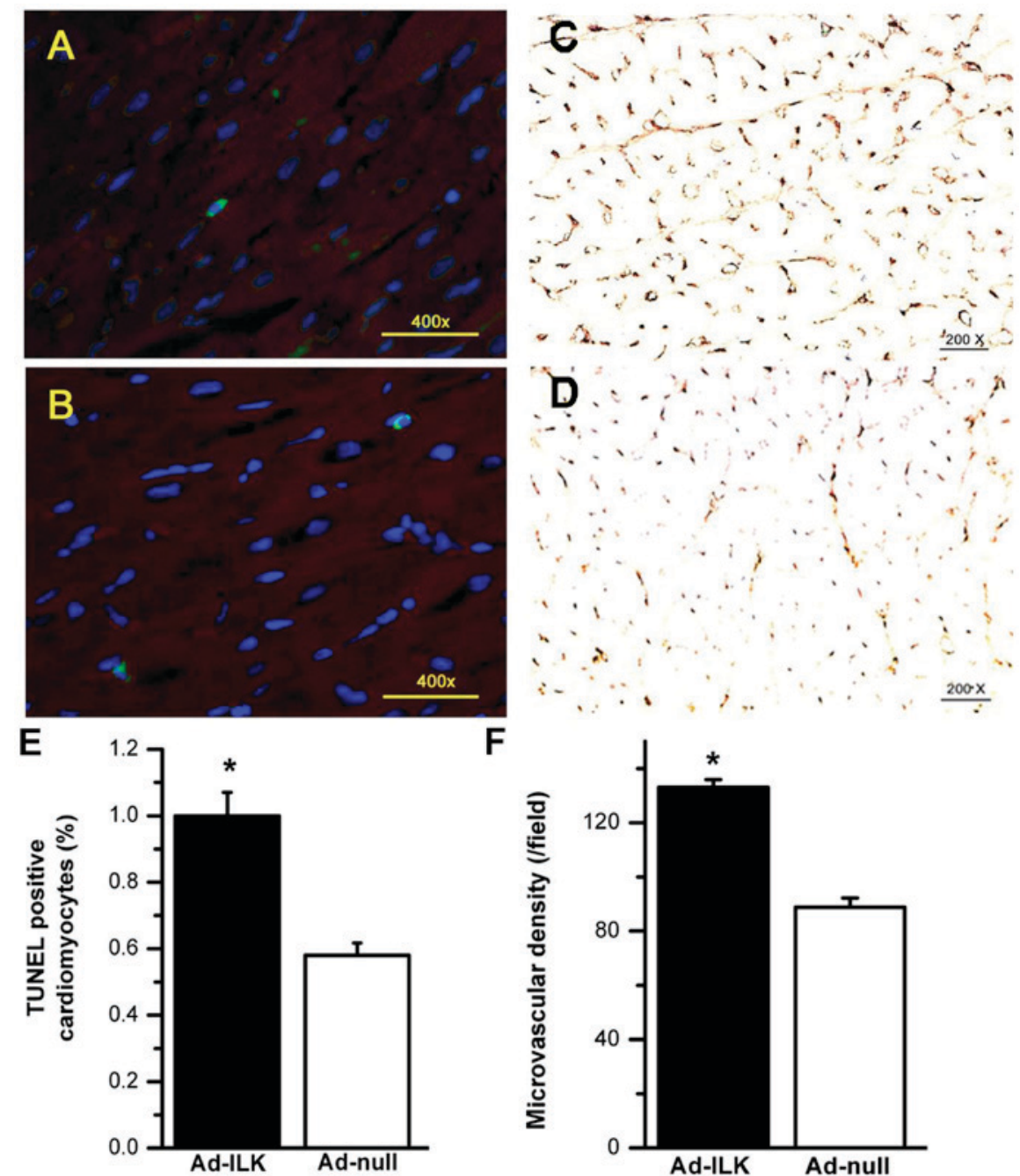

F

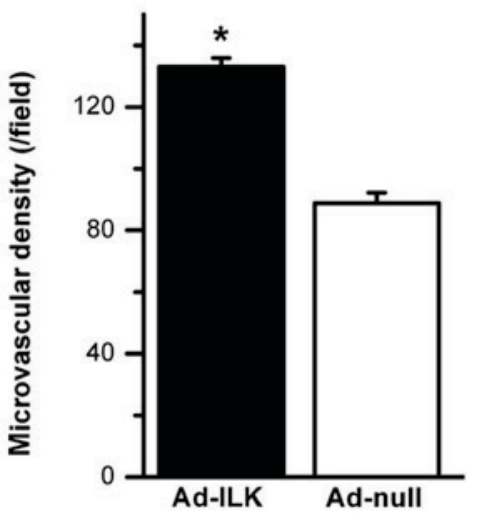

Figure 4. Overexpression of ILK prevents apoptosis and increases microvessel density in the infarct zone. (A and B) Triple-staining with TUNEL (green), anti- $\alpha$-sarcomeric actin antibody (red) and DAPI (blue) in the infarction zone of (A) ad-ILK and (B) ad-null. Scale bars, $50 \mu \mathrm{m}$. (C and D) Microvessel density is measured by frozen sections stained with vWF in the infarct zone of (C) ad-ILK and (D) ad-null group; scale bars, $25 \mu \mathrm{m}$. (E) Quantification of TUNEL-positive cardiomyocytes in the infarct zones of ad-ILK and ad-null animals. $\mathrm{n}=6$ per group. ${ }^{\text {" }} \mathrm{P}<0.01$. (F) Quantification of microvessel density in faction zone of ad-ILK and ad-null animals. $\mathrm{n}=6$ per group. ${ }^{*} \mathrm{P}<0.01$.

The effectiveness of ILK delivery could be ascertained from the studies using AAV1-mediated SERCA-2a gene transfer in preclinical $(14,21,22)$ and clinical studies $(23,24)$, which have shown to boost cardiac contractility and to preserve cardiac function. Traister et al (25) initially revealed increased baseline LV global systolic and diastolic functions in mice with cardiac-specific overexpression of ILK in a SERCA-2a/PLN-dependent mechanism. Thus, ILK serves to link mechanoreception to the dynamic modulation of cardiac contractility in the SERCA-2a/PLN signaling module. Furthermore, ILK provides more potential advantages compared to SERCA-2a based on its SERCA-2a-independent functions. ILK has been confirmed to promote cardiac contractility in the AKT/GSK-3 $\beta$, CamKII, SERCA-2a and $\mathrm{ras} / \mathrm{raf} / \mathrm{MEK}$ pathways. In addition, ILK contributed to angiogenesis and reduced cardiomyocytes apoptosis (26).

Apart from the benefits on cardiomyocytes, ILK also protects fibroblasts from apoptosis $(27,28)$, therefore contributing to the post-infarct healing process by proliferation, collagen synthesis, transformation into myofibroblasts, and reducing the ventricular rupture or aneurysm formation. The present study was consistent with the above discoveries with no aneurysm found in ILK transfer group compared with two pigs in the control group.

Several factors may limit the scope of the present study. First, a more extended period of follow-up would better reflect the progression of heart remodeling and to better contrast the advantages of ILK overexpression relative to the ad-null group. A previous study demonstrated that the improvement in cardiac function after ad-ILK transfection persists up to at least seven weeks in a rat MI model (29). In the present study, although the GFP-positive cells were found in the infarction zone for weeks after gene transfer, we did not directly confirm the expression of ILK. Additionally, apart from detecting ILK and performing histopathology in liver, kidneys, and spleen from ILK-treated animals (data not shown), serum indexes as well as cellular and humoral immunogenicity profiles should be considered to validate the safety of ILK transfer. Furthermore, the expression of the adenoviral vector used in the current study is robust but transient. This approach may be appropriate for short-term, pro-angiogenic responses as in our acute MI model. However, transgene expression levels 
exhibited adenoviral vector peaks within three days and decreases by four weeks (20). In future studies, it may be useful to examine the effectiveness of alternative vectors expressing ILK for much longer periods with lower immunity response, such as recombinant adeno-associated virus.

In conclusion, ILK overexpression by ad-ILK transfer via intracoronary delivery in an acute MI porcine model can preserve global LVEF, improve ventricular remodeling and restore regional perfusion with no signs of toxicology by histopathology assessment, which paves the way for further clinical studies to better define safety and efficacy of this promising approach to ischemic heart disease.

\section{References}

1. Braunwald E: The war against heart failure: The Lancet lecture. Lancet 385: 812-824, 2015.

2. Gajarsa JJ and Kloner RA: Left ventricular remodeling in the post-infarction heart: A review of cellular, molecular mechanisms, and therapeutic modalities. Heart Fail Rev 16: 13-21, 2011.

3. Koitabashi $\mathrm{N}$ and Kass DA: Reverse remodeling in heart failure - mechanisms and therapeutic opportunities. Nat Rev Cardiol 9: 147-157, 2011.

4. Tilemann L, Ishikawa K, Weber T and Hajjar RJ: Gene therapy for heart failure. Circ Res 110: 777-793, 2012.

5. Ding L, Dong L, Chen X, Zhang L, Xu X, Ferro A and Xu B: Increased expression of integrin-linked kinase attenuates left ventricular remodeling and improves cardiac function after myocardial infarction. Circulation 120: 764-773, 2009.

6. Hannigan GE, Leung-Hagesteijn C, Fitz-Gibbon L, Coppolino MG, Radeva G, Filmus J, Bell JC and Dedhar S: Regulation of cell adhesion and anchorage-dependent growth by a new beta 1-integrin-linked protein kinase. Nature 379: 91-96, 1996.

7. Knöll R, Postel R, Wang J, Krätzner R, Hennecke G, Vacaru AM, Vakeel P, Schubert C, Murthy K, Rana BK, et al: Laminin-alpha4 and integrin-linked kinase mutations cause human cardiomyopathy via simultaneous defects in cardiomyocytes and endothelial cells. Circulation 116: 515-525, 2007.

8. White DE, Coutu P, Shi YF, Tardif JC, Nattel S, St Arnaud R, Dedhar S and Muller WJ: Targeted ablation of ILK from the murine heart results in dilated cardiomyopathy and spontaneous heart failure. Genes Dev 20: 2355-2360, 2006.

9. Lee SP, Youn SW, Cho HJ, Li L, Kim TY, Yook HS, Chung JW, Hur J, Yoon CH, Park KW, et al: Integrin-linked kinase, a hypoxia-responsive molecule, controls postnatal vasculogenesis by recruitment of endothelial progenitor cells to ischemic tissue. Circulation 114: 150-159, 2006.

10. Ling L, Bai J, Gu R, Jiang C, Li R, Kang L, Ferro A and $\mathrm{Xu} \mathrm{B}: \mathrm{Sca}^{-} 1^{+}$cardiac progenitor cell therapy with cells overexpressing integrin-linked kinase improves cardiac function after myocardial infarction. Transplantation 95: 1187-1196, 2013.

11. Mao Q, Lin C, Gao J, Liang X, Gao W, Shen L, Kang L and $\mathrm{Xu}$ B: Mesenchymal stem cells overexpressing integrin-linked kinase attenuate left ventricular remodeling and improve cardiac function after myocardial infarction. Mol Cell Biochem 397: 203-214, 2014.

12. Hendel RC, Berman DS, Di Carli MF, Heidenreich PA, Henkin RE, Pellikka PA, Pohost GM and Williams KA; American College of Cardiology Foundation Appropriate Use Criteria Task Force; American Society of Nuclear Cardiology; American College of Radiology; American Heart Association; American Society of Echocardiology; Society of Cardiovascular Computed Tomography; Society for Cardiovascular Magnetic Resonance; Society of Nuclear Medicine: ACCF/ASNC/ACR/AHA/ASE/SCCT/SCMR/SNM 2009 appropriate use criteria for cardiac radionuclide imaging: A report of the American College of Cardiology Foundation appropriate use criteria task force, the American Society of Nuclear Cardiology, the American College of Radiology, the American Heart Association, the American Society of Echocardiography, the Society of Cardiovascular Computed Tomography, the Society for Cardiovascular Magnetic Resonance, and the Society of Nuclear Medicine. J Am Coll Cardiol 53: 2201-2229, 2009.
13. Rutanen J, Rissanen TT, Markkanen JE, Gruchala M, Silvennoinen P, Kivelä A, Hedman A, Hedman M, Heikura T, Ordén MR, et al: Adenoviral catheter-mediated intramyocardial gene transfer using the mature form of vascular endothelial growth factor-D induces transmural angiogenesis in porcine heart. Circulation 109: 1029-1035, 2004.

14. Kawase Y, Ly HQ, Prunier F, Lebeche D, Shi Y, Jin H, Hadri L, Yoneyama R, Hoshino K, Takewa Y, et al: Reversal of cardiac dysfunction after long-term expression of SERCA2a by gene transfer in a pre-clinical model of heart failure. J Am Coll Cardiol 51: 1112-1119, 2008.

15. Thygesen K, Alpert JS, Jaffe AS, Simoons ML, Chaitman BR and White HD; Writing Group on behalf of the Joint ESC/ACCF/AHA/WHF Task Force for the Universal Definition of Myocardial Infarction: Third universal definition of myocardial infarction. Glob Heart 7: 275-295, 2012.

16. Flotats A, Knuuti J, Gutberlet M, Marcassa C, Bengel FM, Kaufmann PA, Rees MR and Hesse B; Cardiovascular Committee of the EANM, the ESCR and the ECNC: Hybrid cardiac imaging: SPECT/CT and PET/CT. A joint position statement by the European Association of Nuclear Medicine (EANM), the European Society of Cardiac Radiology (ESCR) and the European Council of Nuclear Cardiology (ECNC). Eur J Nucl Med Mol Imaging 38: 201-212, 2011.

17. Sutton MG and Sharpe N: Left ventricular remodeling after myocardial infarction: Pathophysiology and therapy. Circulation 101: 2981-2988, 2000.

18. Scimia MC, Gumpert AM and Koch WJ: Cardiovascular gene therapy for myocardial infarction. Expert Opin Biol Ther 14: 183-195, 2014.

19. O'Gara PT, Kushner FG, Ascheim DD, Casey DE Jr, Chung MK, de Lemos JA, Ettinger SM, Fang JC, Fesmire FM, Franklin BA, et al; CF/AHA Task Force: 2013 ACCF/AHA guideline for the management of ST-elevation myocardial infarction: executive summary: a report of the American College of Cardiology Foundation/American Heart Association Task Force on Practice Guidelines. Circulation 127: 529-555, 2013.

20. Hajjar RJ: Potential of gene therapy as a treatment for heart failure. J Clin Invest 123: 53-61, 2013

21. Vejpongsa P and Yeh ETH: Wrestling with heart failure: SUMO-1 to the rescue. Circ Res 114: 1561-1563, 2014.

22. Tilemann L, Lee A, Ishikawa K, Aguero J, Rapti K, SantosGallego C, Kohlbrenner E, Fish KM, Kho C and Hajjar RJ: SUMO-1 gene transfer improves cardiac function in a large-animal model of heart failure. Sci Transl Med 5: 211ra159, 2013.

23. Zsebo K, Yaroshinsky A, Rudy JJ, Wagner K, Greenberg B, Jessup M and Hajjar RJ: Long-term effects of AAV1/SERCA2a gene transfer in patients with severe heart failure: Analysis of recurrent cardiovascular events and mortality. Circ Res 114: 101-108, 2014.

24. Greenberg B, Yaroshinsky A, Zsebo KM, Butler J, Felker GM, Voors AA, Rudy JJ, Wagner K and Hajjar RJ: Design of a phase $2 b$ trial of intracoronary administration of AAV1/SERCA2a in patients with advanced heart failure: The CUPID 2 trial (calcium up-regulation by percutaneous administration of gene therapy in cardiac disease phase 2b). JACC Heart Fail 2: 84-92, 2014.

25. Traister A, Li M, Aafaqi S, Lu M, Arab S, Radisic M, Gross G, Guido F, Sherret J, Verma S, et al: Integrin-linked kinase mediates force transduction in cardiomyocytes by modulating SERCA2a/PLN function. Nat Commun 5: 4533, 2014.

26. Bock-Marquette I, Saxena A, White MD, Dimaio JM and Srivastava D: Thymosin beta4 activates integrin-linked kinase and promotes cardiac cell migration, survival and cardiac repair. Nature 432: 466-472, 2004.

27. Mao Q, Lin CX, Liang XL, Gao JS and Xu B: Mesenchymal stem cells overexpressing integrin-linked kinase attenuate cardiac fibroblast proliferation and collagen synthesis through paracrine actions. Mol Med Rep 7: 1617-1623, 2013.

28. Nho RS, Xia H, Kahm J, Kleidon J, Diebold D and Henke CA: Role of integrin-linked kinase in regulating phosphorylation of Akt and fibroblast survival in type I collagen matrices through a betal integrin viability signaling pathway. J Biol Chem 280: 26630-26639, 2005.

29. Ding L, Dong L, Chen X, Zhang L, Xu X, Ferro A and Xu B: Increased expression of integrin-linked kinase attenuates left ventricular remodeling and improves cardiac function after myocardial infarction. Circulation 120: 764-773, 2009. 